\title{
Qualitative analysis on a predator-prey model with Ivlev functional response
}

\author{
Gaihui Guo ${ }^{1 *}$, Bingfang $\mathrm{Li}^{2}$ and Xiaolin Lin ${ }^{1}$
}

${ }^{\text {"Correspondence: }}$

guogaihui@sust.edu.cn

${ }^{1}$ College of Science, Shaanxi

University of Science and

Technology, Xi'an, 710021, P.R. China

Full list of author information is

available at the end of the article

\begin{abstract}
The diffusive predator-prey model with Ivlev functional response is considered under homogeneous Dirichlet boundary conditions. Firstly, we investigate the bifurcation of positive solutions and derive the multiplicity result for $\gamma$ suitably large. Furthermore, a range of parameters for the uniqueness of positive solutions is described in one dimension. The method we used is based on a comparison principle, Leray-Schauder degree theory, global bifurcation theory and generalized maximum principle.
\end{abstract}

MSC: $35 \mathrm{~K} 57$

Keywords: predator-prey model; Ivlev functional response; bifurcation; uniqueness

\section{Introduction}

In this paper, we are concerned with the following reaction-diffusion system:

$$
\begin{cases}-\Delta u=u(a-u)-v\left(1-e^{-\gamma u}\right), & x \in \Omega, \\ -\Delta v=v\left(c-v+d\left(1-e^{-\gamma u}\right)\right), & x \in \Omega, \\ u=v=0, & x \in \partial \Omega,\end{cases}
$$

where $\Omega$ is a bounded domain in $R^{N}(N \geq 1)$ with smooth boundary $\partial \Omega, u, v$ represent the population density of prey and predator, respectively. $a$ is the natural growth rate of prey, $d$ is the conversion rate of a consumed prey to a predator, $\gamma$ is the efficiency of the predator for capturing prey. $a, c, d$ and $\gamma$ are constants with $a, d$ positive and $\gamma$ non-negative; $c$ may change sign and $c>0$ indicates the predator has other food sources. This is a prey dependent predator-prey model with the Ivlev-type functional response $1-e^{-\gamma u}$, which was originally introduced by Ivlev in [1].

The predator-prey model has long been one of the dominant themes due to its universal existence and importance. Both ecologists and mathematicians are interested in the Ivlevtype predator-prey model; see [2-9] for example. The existence and uniqueness of limit cycle for the Ivlev response predator-prey system were studied in $[2,3]$. The conditions for the permanence of the Ivlev system and the existence and stability of a positive periodic solution were investigated in [4]. The dynamical behavior analysis of the Ivlev response predator-prey systems was discussed in [1, 5-7]. To our knowledge, there are few works on such a type of functional response in the reaction-diffusion system. Under Neumann boundary conditions, the spatial pattern formation of the model was carried out by using Hopf bifurcation in [8]. Under Dirichlet boundary conditions, a sufficient and necessary condition for the existence of positive solutions to the model was obtained in [9].

(c) 2013 Guo et al.; licensee Springer. This is an Open Access article distributed under the terms of the Creative Commons Attribution License (http://creativecommons.org/licenses/by/2.0), which permits unrestricted use, distribution, and reproduction in any medium, provided the original work is properly cited. 
Now, we introduce some notations and basic facts which will be often used later. Let $X$ be the Banach space

$$
X=\left\{u \in C^{1}(\bar{\Omega}): u(x)=0, x \in \partial \Omega\right\} .
$$

Let $P=\left\{u \in X: u>0\right.$ in $\Omega$ and $\partial_{v} u<0$ on $\left.\partial \Omega\right\}$ be the usual positive cone in $X$, where $v$ is the outward unit normal vector on $\partial \Omega$ and $\partial_{v}=\partial / \partial v$. For $q(x) \in C(\bar{\Omega})$, let $\lambda_{1}(q)<\lambda_{2}(q) \leq$ $\lambda_{3}(q) \leq \cdots$ be all eigenvalues of the following problem:

$$
-\Delta \phi+q(x) \phi=\lambda \phi \quad \text { in } \Omega, \quad \phi=0 \quad \text { on } \partial \Omega .
$$

It follows from [10] that $\lambda_{1}(q)$ is simple and $\lambda_{1}(q)$ is strictly increasing in the sense that $q_{1} \leq q_{2}$ and $q_{1} \not \equiv q_{2}$ implies $\lambda_{1}\left(q_{1}\right)<\lambda_{1}\left(q_{2}\right)$. When $q(x) \equiv 0$, we denote $\lambda_{i}(0)$ by $\lambda_{i}$ for the sake of convenience. Moreover, we denote by $\Phi_{1}(>0)$ the eigenfunction corresponding to $\lambda_{1}$ with normalization $\left\|\Phi_{1}\right\|_{2}^{2}=1$.

For any $a>\lambda_{1}$, it is well known that the problem

$$
-\Delta u=(a-u) u \quad \text { in } \Omega, \quad u=0 \quad \text { on } \partial \Omega
$$

has a unique positive solution which we denote by $\theta_{a}$. It is also known that the mapping $a \rightarrow \theta_{a}$ is strictly increasing, continuously differentiable in $\left(\lambda_{1}, \infty\right)$, and that $\theta_{a} \rightarrow 0$ uniformly on $\bar{\Omega}$ as $a \rightarrow \lambda_{1}$. Moreover, $0<\theta_{a}<a$ in $\Omega$. Therefore, if $a>\lambda_{1}$, then (1.1) has a semi-trivial solution $\left(\theta_{a}, 0\right)$. Similar results hold with respect to another semi-trivial solution $\left(0, \theta_{c}\right)$ whenever $c>\lambda_{1}$. We extend the definition of $\theta_{c}$ by taking $\theta_{c} \equiv 0$ if $c \leq \lambda_{1}$.

This work mainly aims at establishing the existence, multiplicity and uniqueness of positive solutions to (1.1). More precisely, a sufficient and necessary condition for the existence of positive solutions is given when $c \leq \lambda_{1}$, and when $c>\lambda_{1}$, the multiplicity of positive solutions is obtained under the assumption that $\gamma$ is suitably large. If $\gamma$ is suitably small, then we get the uniqueness of positive solutions in one dimension.

The rest of this paper is organized as follows. In Section 2, by calculating the indices of fixed points, we obtain sufficient conditions for the existence of positive solutions to (1.1). In Section 3, by investigating the bifurcation of positive solutions emanating from the semi-trivial solution $\left(\theta_{a}, 0 ; c\right)$, we give a sufficient and necessary condition for the existence of positive solutions to (1.1) and establish the multiplicity result of positive solutions when $\gamma$ is suitably large. In Section 4 , assuming that $\Omega=(p, q)$ is an interval, we find that (1.1) has at most one positive solution when $\gamma^{2}(c+d) \leq 2$.

\section{The existence of positive solutions}

In this section, we establish the existence and nonexistence of positive solutions to (1.1). A necessary condition and a priori estimate are firstly given. The proofs are standard and will be omitted.

Lemma 2.1 If (1.1) has a positive solution, then we have

$$
a>\lambda_{1} \text { and } c+d>\lambda_{1} \text {. }
$$


Lemma 2.2 Assume that $(u, v)$ is a positive solution of $(1.1)$. Then $(u, v)$ satisfies

$$
0<u<\theta_{a}<a, \quad 0<v<\theta_{c+d}<c+d \quad \text { in } \Omega .
$$

In addition, $v>\theta_{c}$ if $c>\lambda_{1}$.

Next, we set up the fixed point index theory for later use. Let $E$ be a real Banach space and $W$ be a closed convex set of $E$. For $y \in W$, define $W_{y}=\{x \in E: y+\gamma x \in W$ for some $\gamma>0\}$ and $S_{y}=\left\{x \in \bar{W}_{y}:-x \in \bar{W}_{y}\right\}$. Let $F: W \rightarrow W$ be a compact operator with a fixed point $y \in W$, and denote by $L$ the Fréchet derivative of $F$ at $y$. Then $L$ maps $\bar{W}_{y}$ into itself. We say that $L$ has property $\alpha$ on $\bar{W}_{y}$ if there exist $t \in(0,1)$ and $w \in \bar{W}_{y} \backslash S_{y}$ such that $w-t L w \in S_{y}$.

For an open subset $U \subset W$, define $\operatorname{index}_{W}(F, U)=\operatorname{index}(F, U, W)=\operatorname{deg}_{W}(I-F, U, 0)$, where $I$ is the identity map. If $y$ is an isolated fixed point of $F$, then the fixed point index of $F$ at $y$ in $W$ is defined by $\operatorname{index}_{W}(F, y)=\operatorname{index}(F, U(y), W)$, where $U(y)$ is a small open neighborhood of $y$ in $W$.

Lemma 2.3 (See [11]) Assume that I - L is invertible on $\bar{W}_{y}$.

(i) If $L$ has property $\alpha$ on $\bar{W}_{y}$, then $\operatorname{index}_{W}(F, y)=0$.

(ii) If $L$ does not have property $\alpha$ on $\bar{W}_{y}$, then $\operatorname{index}_{W}(F, y)=(-1)^{\sigma}$, where $\sigma$ is the sum of algebra multiplicities of the eigenvalues of $L$ which are greater than 1 .

Denote by $r(L)$ the spectral radius of a linear operator $L$.

Lemma 2.4 (See [12]) Let $q(x) \in C(\bar{\Omega})$ and let $M$ be a positive constant such that $-q(x)+$ $M>0$ on $\bar{\Omega}$. Then we have the following conclusions:

(i) $\lambda_{1}(q(x))<0 \Longrightarrow r\left[(-\Delta+M)^{-1}(-q(x)+M)\right]>1$;

(ii) $\lambda_{1}(q(x))>0 \Longrightarrow r\left[(-\Delta+M)^{-1}(-q(x)+M)\right]<1$;

(iii) $\lambda_{1}(q(x))=0 \Longrightarrow r\left[(-\Delta+M)^{-1}(-q(x)+M)\right]=1$.

Now we introduce the following notations:

(i) $E:=C_{0}(\bar{\Omega}) \oplus C_{0}(\bar{\Omega})$, where $C_{0}(\bar{\Omega})=\{u \in C(\Omega): u(x)=0$ on $\partial \Omega\}$;

(ii) $W:=P_{1} \oplus P_{1}$, where $P_{1}=\left\{u \in C_{0}(\bar{\Omega}): u(x)>0\right.$ in $\left.\Omega\right\}$;

(iii) $\mathcal{D}:=\{(u, v) \in E: u<a, v<c+d\}$;

(iv) $\mathcal{D}^{\prime}:=($ int $\mathcal{D}) \cap W$.

From Lemma 2.2, we see that all the non-negative solutions of (1.1) must be in $\mathcal{D}$. For any $\tau \in[0,1]$, define a positive compact operator $\mathcal{A}_{\tau}: \mathcal{D}^{\prime} \rightarrow W$ by

$$
\mathcal{A}_{\tau}(u, v)=(-\Delta+M)^{-1}\left(\begin{array}{c}
\tau u(a-u)-v\left(1-e^{-\gamma u}\right)+M u \\
\tau v\left(c-v+d\left(1-e^{-\gamma u}\right)\right)+M v
\end{array}\right),
$$

where $M$ is large such that $\max \{a+(c+d) \gamma, d\}<M$. It follows from the standard elliptic regularity theory that $\mathcal{A}_{\tau}$ is a completely continuous operator. Observe that (1.1) has a positive solution in $W$ if and only if $\mathcal{A}:=\mathcal{A}_{1}$ has a positive fixed point in $\mathcal{D}^{\prime}$. If $a, c>\lambda_{1}$, then $(0,0),\left(\theta_{a}, 0\right),\left(0, \theta_{c}\right)$ are the only non-negative fixed points of $\mathcal{A}$ which are not positive. The corresponding indices in $W$ can be calculated in the following lemmas.

Lemma 2.5 Assume that $a>\lambda_{1}$. 
(i) $\operatorname{Index}_{W}\left(\mathcal{A}, \mathcal{D}^{\prime}\right)=1$.

(ii) If $c \neq \lambda_{1}$, then $\operatorname{index}_{W}(\mathcal{A},(0,0))=0$.

(iii) If $c>\lambda_{1}\left(-d\left(1-e^{-\gamma \theta_{a}}\right)\right)$, then $\operatorname{index}_{W}\left(\mathcal{A},\left(\theta_{a}, 0\right)\right)=0$.

(iv) If $c<\lambda_{1}\left(-d\left(1-e^{-\gamma \theta_{a}}\right)\right)$, then $\operatorname{index}_{W}\left(\mathcal{A},\left(\theta_{a}, 0\right)\right)=1$.

Proof (i) Since $\mathcal{A}_{\tau}$ has no fixed point on $\mathcal{D}^{\prime}$, the degree $\operatorname{deg}_{W}\left(I-\mathcal{A}_{\tau}, \mathcal{D}^{\prime}, 0\right)$ is well defined. It is easy to see that all fixed points of $\mathcal{A}_{\tau}$ are in $\mathcal{D}^{\prime}$. Therefore, by the homotopy invariance of degree, $\operatorname{deg}_{W}\left(I-\mathcal{A}_{\tau}, \mathcal{D}^{\prime}, 0\right)$ is independent of $\tau$. Then

$$
\begin{aligned}
\operatorname{index}_{W}\left(\mathcal{A}, \mathcal{D}^{\prime}\right) & =\operatorname{deg}_{W}\left(I-\mathcal{A}, \mathcal{D}^{\prime}, 0\right)=\operatorname{deg}_{W}\left(I-\mathcal{A}_{\tau}, \mathcal{D}^{\prime}, 0\right) \\
& =\operatorname{deg}_{W}\left(I-\mathcal{A}_{0}, \mathcal{D}^{\prime}, 0\right)
\end{aligned}
$$

Observing that (1.1) has only the trivial solution $(0,0)$ when $\tau=0$, we have

$$
\operatorname{deg}_{W}\left(I-\mathcal{A}_{0}, \mathcal{D}^{\prime}, 0\right)=\operatorname{index}_{W}\left(\mathcal{A}_{0},(0,0)\right)
$$

Let $L=\mathcal{A}_{0}^{\prime}(0,0)$. Then

$$
L=(-\Delta+M)^{-1}\left(\begin{array}{cc}
M & 0 \\
0 & M
\end{array}\right)
$$

It is easy to see that $r(L)<1$ by Lemma 2.4. This implies that $I-L$ is invertible on $\bar{W}_{(0,0)}$ and $L$ does not have property $\alpha$ on $\bar{W}_{(0,0)}$. By Lemma 2.3 , index $\operatorname{Lex}_{W}\left(\mathcal{A}_{0},(0,0)\right)=1$. It follows from (2.1) and (2.2) that $\operatorname{index}_{W}\left(\mathcal{A}, \mathcal{D}^{\prime}\right)=1$.

(ii) It is easy to observe that $W_{(0,0)}=W, S_{(0,0)}=\{(0,0)\}$. Let $L=\mathcal{A}^{\prime}(0,0)$. Then

$$
L=(-\Delta+M)^{-1}\left(\begin{array}{cc}
a+M & 0 \\
0 & c+M
\end{array}\right) \text {. }
$$

Assume that $L(\xi, \eta)=(\xi, \eta)$ for some $(\xi, \eta) \in \bar{W}_{(0,0)}$. Then

$$
-\Delta \xi=a \xi, \quad-\Delta \eta=c \eta, \quad x \in \Omega, \quad \xi=\eta=0, \quad x \in \partial \Omega
$$

Since $a \neq \lambda_{1}, c \neq \lambda_{1}$, we have $\xi=\eta=0$. Thus $I-L$ is invertible on $\bar{W}_{(0,0)}$.

Note that $a>\lambda_{1}$. By Lemma 2.4, we know that $r_{a}:=r\left[(-\Delta+M)^{-1}(a+M)\right]>1$, and $r_{a}$ is the principal eigenvalue of the operator $(-\Delta+M)^{-1}(a+M)$ with the corresponding eigenfunction $\phi>0$. Set $t_{0}=1 / r_{a}$. Then $t_{0} \in(0,1)$ and $\left(I-t_{0} L\right)(\phi, 0)=(0,0) \in S_{(0,0)}$. This shows that $L$ has property $\alpha$. By Lemma 2.3 , $\operatorname{index}_{W}(\mathcal{A},(0,0))=0$.

(iii) Let $y=\left(\theta_{a}, 0\right)$. Then $W_{y}=\{(\xi, \eta) \in E, \eta \geq 0\}, S_{y}=\left\{(\xi, 0): \xi \in C_{0}^{1}(\bar{\Omega})\right\}$. Set $L=$ $\mathcal{A}^{\prime}\left(\theta_{a}, 0\right)$. Then we have

$$
L=(-\Delta+M)^{-1}\left(\begin{array}{cc}
a-2 \theta_{a}+M & -\left(1-e^{-\gamma \theta_{a}}\right) \\
0 & c+d\left(1-e^{-\gamma \theta_{a}}\right)+M
\end{array}\right) \text {. }
$$


Assume that $L(\xi, \eta)=(\xi, \eta)$ for some $(\xi, \eta) \in \bar{W}_{y}$. Then

$$
\begin{cases}-\Delta \xi-\left(a-2 \theta_{a}\right) \xi+\left(1-e^{-\gamma \theta_{a}}\right) \eta=0, & x \in \Omega, \\ -\Delta \eta-\left(c+d\left(1-e^{-\gamma \theta_{a}}\right)\right) \eta=0, & x \in \Omega, \\ \xi=\eta=0, & x \in \partial \Omega .\end{cases}
$$

If $c \neq \lambda_{1}\left(-d\left(1-e^{-\gamma \theta_{a}}\right)\right)$, then $\eta=0$. And we further have $\xi=0$. Thus $I-L$ is invertible on $\bar{W}_{y}$.

Note that $c>\lambda_{1}\left(-d\left(1-e^{-\gamma \theta_{a}}\right)\right)$. By Lemma 2.4, we know that $r_{c}:=r\left[(-\Delta+M)^{-1}(c+d(1-\right.$ $\left.\left.\left.e^{-\gamma \theta_{a}}\right)+M\right)\right]>1$ is the principal eigenvalue of the operator $(-\Delta+M)^{-1}\left(c+d\left(1-e^{-\gamma \theta_{a}}\right)+M\right)$ with the corresponding eigenfunction $\psi>0$. Set $t_{0}=1 / r_{c}$. Then $t_{0} \in(0,1),(0, \psi) \in \bar{W}_{y} \backslash S_{y}$ and

$$
\begin{aligned}
\left(I-t_{0} L\right)\left(\begin{array}{l}
0 \\
\psi
\end{array}\right) & =\left(\begin{array}{c}
t_{0}(-\Delta+M)^{-1}\left(1-e^{-\gamma \theta_{a}}\right) \psi \\
\psi-t_{0}(-\Delta+M)^{-1}\left(c+d\left(1-e^{-\gamma \theta_{a}}\right)+M\right) \psi
\end{array}\right) \\
& =\left(\begin{array}{c}
t_{0}(-\Delta+M)^{-1}\left(1-e^{-\gamma \theta_{a}}\right) \psi \\
0
\end{array}\right) \in S_{y} .
\end{aligned}
$$

This shows that $L$ has property $\alpha$. By Lemma 2.3, index $W\left(\mathcal{A},\left(\theta_{a}, 0\right)\right)=0$.

(iv) Since $c \neq \lambda_{1}\left(-d\left(1-e^{-\gamma \theta_{a}}\right)\right), I-L$ is invertible on $\bar{W}_{y}$. We claim that $L$ does not have property $\alpha$ on $\bar{W}_{y}$. Note that $c<\lambda_{1}\left(-d\left(1-e^{-\gamma \theta_{a}}\right)\right)$. By Lemma 2.4, we know that $r_{c}:=r\left[(-\Delta+M)^{-1}\left(c+d\left(1-e^{-\gamma \theta a}\right)+M\right)\right]<1$. Suppose that $L$ has property $\alpha$ on $\bar{W}_{y}$. Then there exist $t_{1} \in(0,1)$ and $\left(\phi_{1}, \psi_{1}\right) \in \bar{W}_{y} \backslash S_{y}$ such that $\left(I-t_{1} L\right)\left(\phi_{1}, \psi_{1}\right) \in S_{y}$. Therefore,

$$
\psi_{1}-t_{1}(-\Delta+M)^{-1}\left(c+d\left(1-e^{-\gamma \theta_{a}}\right)+M\right) \psi_{1}=0
$$

Since $\psi_{1}>0,1 / t_{1}>1$ is a principal eigenvalue of the operator $(-\Delta+M)^{-1}(c+d(1-$ $\left.\left.e^{-\gamma \theta_{a}}\right)+M\right)$, which is contradiction to $r_{c}<1$. Thus $L$ does not have property $\alpha$ on $\bar{W}_{y}$. By Lemma 2.3, index $W\left(\mathcal{A},\left(\theta_{a}, 0\right)\right)=(-1)^{\sigma}$, where $\sigma$ is the sum of the multiplicities of all real eigenvalues of $L$ which are greater than 1 .

Assume that $\lambda>1$ is an eigenvalue of $L$ with a corresponding eigenfunction $(\xi, \eta)$. Then simple calculations yield

$$
\begin{cases}-\Delta \xi+M \xi=\frac{1}{\lambda}\left[\left(a-2 \theta_{a}+M\right) \xi-\left(1-e^{-\gamma \theta_{a}}\right) \eta\right]=0, & x \in \Omega, \\ -\Delta \eta+M \eta=\frac{1}{\lambda}\left[c+d\left(1-e^{-\gamma \theta_{a}}\right)\right] \eta=0, & x \in \Omega, \\ \xi=\eta=0, & x \in \partial \Omega .\end{cases}
$$

If $\eta \neq 0$, then from the second equation of (2.3), we obtain

$$
0=\lambda_{1}\left(M(1-1 / \lambda)-1 / \lambda\left(c+d\left(1-e^{-\gamma \theta_{a}}\right)\right)\right)>\lambda_{1}\left(-d\left(1-e^{-\gamma \theta_{a}}\right)\right)-c>0 .
$$

This contradiction shows that $\eta=0$. Thus $\xi \neq 0$. From the first equation of (2.3), we have

$$
0=\lambda_{1}\left(M(1-1 / \lambda)-1 / \lambda\left(a-2 \theta_{a}\right)\right)>\lambda_{1}\left(-a+2 \theta_{a}\right)>0 .
$$

This contradiction shows that $L$ has no eigenvalues being greater than 1 . Consequently, $\sigma=0$. Hence, $\operatorname{index}_{W}\left(\mathcal{A},\left(\theta_{a}, 0\right)\right)=1$. 
Similarly, we can obtain the following lemma.

Lemma 2.6 Assume that $c>\lambda_{1}$.

(i) If $a>\lambda_{1}\left(\gamma \theta_{c}\right)$, then index $\operatorname{de}_{W}\left(\mathcal{A},\left(0, \theta_{c}\right)\right)=0$.

(ii) If $a<\lambda_{1}\left(\gamma \theta_{c}\right)$, then $\operatorname{index}_{W}\left(\mathcal{A},\left(0, \theta_{c}\right)\right)=1$.

By the additivity property of the index, the existence of positive solutions to (1.1) is obtained.

Theorem 2.1 (i) If $a>\lambda_{1}\left(\gamma \theta_{c}\right), c>\lambda_{1}$, then (1.1) has at least a positive solution.

(ii) If $a>\lambda_{1}, \lambda_{1}\left(-d\left(1-e^{-\gamma \theta_{a}}\right)\right)<c<\lambda_{1}$, then (1.1) has at least a positive solution.

Proof Argue by contradiction. Suppose that (1.1) has no positive solution.

(i) If $a>\lambda_{1}$ and $\lambda_{1}\left(-d\left(1-e^{-\gamma \theta_{a}}\right)\right)<c<\lambda_{1}$, then by Lemma 2.5 and the additivity property of the index, we have

$$
1=\operatorname{index}_{W}\left(\mathcal{A}, \mathcal{D}^{\prime}\right)=\operatorname{index}_{W}(\mathcal{A},(0,0))+\operatorname{index}_{W}\left(\mathcal{A},\left(\theta_{a}, 0\right)\right)=0 .
$$

The contradiction implies that (1.1) has at least a positive solution in $\mathcal{D}^{\prime}$.

(ii) If $a>\lambda_{1}\left(\gamma \theta_{c}\right)$ and $c>\lambda_{1}$, then by Lemmas 2.5, 2.6 and the additivity property of the index, we have

$$
1=\operatorname{index}_{W}\left(\mathcal{A}, \mathcal{D}^{\prime}\right)=\operatorname{index}_{W}(\mathcal{A},(0,0))+\operatorname{index}_{W}\left(\mathcal{A},\left(\theta_{a}, 0\right)\right)+\operatorname{index}_{W}\left(\mathcal{A},\left(0, \theta_{c}\right)\right)=0 .
$$

The contradiction implies that (1.1) has at least a positive solution in $\mathcal{D}^{\prime}$.

\section{Bifurcation and multiplicity of positive solutions}

In this section, by discussing the bifurcations of positive solutions by using $a$ and $c$ as the main bifurcation parameters, respectively, we establish the multiplicity of positive solutions when $\gamma$ is suitably large. First, we show that (1.1) has no positive solution when $c$ is sufficiently large.

Lemma 3.1 If (1.1) has a positive solution, then there exists a sufficiently large constant $M>0$ such that $\lambda_{1}\left(-d\left(1-e^{-\gamma \theta_{a}}\right)\right)<c<M$.

Proof Suppose that (1.1) has a positive solution $(u, v)$. Then by Lemma 2.2, we have

$$
c=\lambda_{1}\left(\nu-d\left(1-e^{-\gamma u}\right)\right)>\lambda_{1}\left(-d\left(1-e^{-\gamma \theta_{a}}\right)\right) .
$$

Moreover, since the function $f(s)=\left(1-e^{-s}\right) / s$ is strictly decreasing with respect to $s>0$, considering the equation of $u$, we find

$$
a=\lambda_{1}\left(u+\gamma \nu \frac{1-e^{-\gamma u}}{\gamma u}\right)>\lambda_{1}\left(\frac{1-e^{-\gamma \theta_{a}}}{\theta_{a}} \theta_{c}\right) .
$$

Choose $c$ large enough. Then for fixed $a$, we have $\lambda_{1}\left(\left(1-e^{-\gamma \theta_{a}}\right) / \theta_{a} \theta_{c}\right)>a$, which is a contradiction to (3.1). 
Fixing $a>\lambda_{1}$ and taking $c$ as a bifurcation parameter, we shall obtain positive solutions bifurcating from the semi-trivial solution $\left(\theta_{a}, 0\right)$.

Theorem 3.1 Assume that $a>\lambda_{1}$. Let $\tilde{c}=\lambda_{1}\left(-d\left(1-e^{-\gamma \theta_{a}}\right)\right)$. Then $\left(\theta_{a}, 0 ; \tilde{c}\right)$ is a bifurcation point of the positive solution to (1.1). Moreover, there exists a constant $\delta>0$ and a $C^{1}$-curve $(u(s), v(s) ; c(s)):(0, \delta) \rightarrow X \times X \times R$ such that

(i) $(u(s), v(s))$ is a positive solution of $(1.1)$ with $c=c(s)$ for each $s \in(0, \delta]$ and

$$
u(s)=\theta_{a}+s(\tilde{\phi}+\phi(s)), \quad v(s)=s(\tilde{\psi}+\psi(s))
$$

where $\tilde{\psi}$ is a positive eigenfunction corresponding to $\tilde{c}$ with $\int_{\Omega} \tilde{\psi}^{2} \mathrm{~d} x=1$,

$\tilde{\phi}=\left(\Delta+a-2 \theta_{a}\right)^{-1}\left(\left(1-e^{-\gamma \theta_{a}}\right) \tilde{\psi}\right)<0,(\phi, \psi) \in Z, Z \oplus \operatorname{span}\{(\tilde{\phi}, \tilde{\psi})\}=X \times X ;$

(ii) $c(0)=\tilde{c},(u(0), v(0))=\left(\theta_{a}, 0\right)$, and $\phi(0)=\psi(0)=0$.

By the classic Crandall-Rabinowitz bifurcation theorem in [13], one can obtain Theorem 3.1 easily. So the proof is omitted here. One can refer to $[14,15]$ for similar arguments. Now we state a sufficient condition for the existence of positive solutions as follows.

Theorem 3.2 If the following relationship holds:

$$
a>\lambda_{1}\left(\gamma \theta_{c}\right) \text { and } c>\lambda_{1}\left(-d\left(1-e^{-\gamma \theta_{a}}\right)\right)
$$

then (1.1) has at least a positive solution.

Proof Fixing $a>\lambda_{1}$ and taking $c$ as the main bifurcation parameter, we can obtain a supercritical bifurcating branch of positive solutions to (1.1), which emanates from the semitrivial solution $\left(\theta_{a}, 0\right)$ at the value of $\tilde{c}=\lambda_{1}\left(-d\left(1-e^{-\gamma \theta_{a}}\right)\right)$. The existence was given in Theorem 3.1. It suffices to show the bifurcation direction. To this end, substitute $(u(s), v(s) ; c(s))$ given by (3.2) into the second equation of (1.1), divide by $s$, differentiate with respect to $s$ and set $s=0$, which leads to

$$
-\Delta \psi^{\prime}(0)=\left[\tilde{c}+d\left(1-e^{-\gamma \theta_{a}}\right)\right] \psi^{\prime}(0)+\left[c^{\prime}(0)-\tilde{\psi}+d \gamma e^{-\gamma \theta_{a}} \tilde{\psi}\right] \tilde{\psi} .
$$

Now, multiply by $\tilde{\psi}$ and integrate over $\Omega$ to get

$$
-\int_{\Omega} \psi^{\prime}(0) \Delta \tilde{\psi} \mathrm{d} x=\int_{\Omega} \tilde{\psi}\left[\tilde{c}+d\left(1-e^{-\gamma \theta_{a}}\right)\right] \psi^{\prime}(0) \mathrm{d} x+\int_{\Omega}\left[c^{\prime}(0)-\tilde{\psi}+d \gamma e^{-\gamma \theta_{a}}\right] \tilde{\psi}^{2} \mathrm{~d} x
$$

Hence, we have

$$
c^{\prime}(0)=\int_{\Omega} \tilde{\psi}^{3} \mathrm{~d} x-d \gamma \int_{\Omega} e^{-\gamma \theta_{a}} \tilde{\phi} \tilde{\psi}^{2} \mathrm{~d} x
$$

Noting that $\tilde{\phi}<0$, we obtain $c^{\prime}(0)>0$, which shows that the bifurcating branch is supercritical.

In the following, we shall investigate the global structure of bifurcation solutions given by Theorem 3.1 and then the relationship in (3.2) can be obtained. By the global bifurcation theorem in [10], we can extend the local bifurcation positive solution to the global one. One 
can see $[15,16]$ for similar arguments. From Lemma 3.1, we know that the parameter $c$ is bounded. And from a priori estimates given by Lemma 2.2, it follows that $\|u\|_{\infty}$ and $\|v\|_{\infty}$ are also bounded. Hence, we claim that the continuum of positive solutions bifurcating from $\left(\theta_{a}, 0 ; \tilde{c}\right)$ cannot remain in the interior of $P \times P$, which implies that there must exist $\hat{c}(>\tilde{c})$, at which one of the components of the continuum of positive solutions vanishes. Let $c_{n}$ be a strictly increasing sequence converging to $\hat{c} \in(\tilde{c}, \infty)$ for which (1.1) has at least a positive solution $\left(u_{n}, v_{n}\right)$ such that $u_{n} \rightarrow 0$ or $v_{n} \rightarrow 0$ as $n \rightarrow \infty$. If we denote by $(\hat{u}, \hat{v})$ the limit of $\left(u_{n}, v_{n}\right)$ as $n \rightarrow \infty$, then we have the following three cases:
(i) $(\hat{u}, \hat{v})=\left(0, \theta_{\hat{c}}\right)$,
(ii) $(\hat{u}, \hat{v})=\left(\theta_{a}, 0\right)$,
(iii) $(\hat{u}, \hat{v})=(0,0)$.

Since $(0,0)$ is non-degenerate, (iii) is excluded. Set $\tilde{v}_{n}=v_{n} /\left\|v_{n}\right\|_{\infty}$. Then $\tilde{v}_{n}$ satisfies

$$
-\Delta \tilde{v}_{n}=\left(c_{n}-v_{n}+d\left(1-e^{-\gamma u_{n}}\right)\right) \tilde{v}_{n}, \quad\left\|\tilde{v}_{n}\right\|_{\infty}=1 \quad \text { in } \Omega, \quad \tilde{v}_{n}=0 \quad \text { on } \partial \Omega .
$$

Letting $n \rightarrow \infty$, we have

$$
-\Delta \tilde{v}=\left(\hat{c}+d\left(1-e^{-\gamma \theta_{a}}\right)\right) \tilde{v}, \quad\|\tilde{v}\|_{\infty}=1 \quad \text { in } \Omega, \quad \tilde{v}=0 \quad \text { on } \partial \Omega .
$$

It follows from $\tilde{v} \in P-\{0\}$ that $\hat{c}=\lambda_{1}\left(-d\left(1-e^{-\gamma \theta a}\right)\right)=\tilde{c}$, which contradicts the global bifurcation theorem. (ii) is also excluded. Hence, we know that (i) holds true. Set $\tilde{u}_{n}=u_{n} /\left\|u_{n}\right\|_{\infty}$. Then $\tilde{u}_{n}$ satisfies

$$
-\Delta \tilde{u}_{n}=\left(a-u_{n}-v_{n} \frac{1-e^{-\gamma u_{n}}}{u_{n}}\right) \tilde{u}_{n}, \quad\left\|\tilde{u}_{n}\right\|_{\infty}=1 \quad \text { in } \Omega, \quad \tilde{u}_{n}=0 \quad \text { on } \partial \Omega .
$$

Letting $n \rightarrow \infty$, we have

$$
-\Delta \tilde{u}=\left(a-\gamma \theta_{\hat{c}}\right) \tilde{u}, \quad\|\tilde{u}\|_{\infty}=1 \quad \text { in } \Omega, \quad \tilde{u}=0 \quad \text { on } \partial \Omega .
$$

It follows from $\tilde{u} \in P-\{0\}$ that $a=\lambda_{1}\left(\gamma \theta_{\hat{c}}\right)$. Now, we know that $\hat{c}$ is uniquely determined by $a=\lambda_{1}\left(\gamma \theta_{c}\right)$. Observe that $c<\hat{c}$ implies that $a>\lambda_{1}\left(\gamma \theta_{c}\right)$. Hence, if $a>\lambda_{1}\left(\gamma \theta_{c}\right)$ and $c>\tilde{c}$, then (1.1) has at least a positive solution.

Remark 3.1 The condition (3.3) is better than those obtained in Theorem 2.1. In particular, (3.3) includes the case $c=\lambda_{1}$. Note that Theorem 2.1 tells us nothing when $c=\lambda_{1}$. Since $I-L$ is not invertible on $\bar{W}_{(0,0)}$ when $c=\lambda_{1}$, Lemma 2.3 is not satisfied and so we cannot use it to get the index of the fixed point $(0,0)$.

Fix $a>\lambda_{1}$ and take $c$ as the bifurcation parameter, then by the proof of Theorem 3.2, we can obtain a supercritical bifurcating branch from the point $\left(\lambda_{1}\left(-d\left(1-e^{-\gamma \theta_{a}}\right)\right) ; \theta_{a}, 0\right)$. Moreover, resorting to the global bifurcation theory, we get the maximal continuum of positive solutions, which tells us the range of the parameter $c$ is $\lambda_{1}\left(-d\left(1-e^{-\gamma \theta a}\right)\right)<c<\hat{c}$, where $\hat{c}$ is determined uniquely by $a=\lambda_{1}\left(\gamma \theta_{\hat{c}}\right)$. Hence, a sufficient and necessary condition for the existence of positive solutions can be stated in the following remark.

Remark 3.2 Assume $a>\lambda_{1}$. Then (1.1) has a positive solution if and only if $\lambda_{1}(-d(1-$ $\left.\left.e^{-\gamma \theta_{a}}\right)\right)<c<\hat{c}$, where $\hat{c}$ is determined uniquely by $a=\lambda_{1}\left(\gamma \theta_{\hat{c}}\right)$. 
In the case $c>\lambda_{1}$, Theorem 3.2 tells us that if $a>\lambda_{1}\left(\gamma \theta_{c}\right)$, then (1.1) has at least a positive solution. A natural question is whether (1.1) has a positive solution if $a \in\left(\lambda_{1}, \lambda_{1}\left(\gamma \theta_{c}\right)\right]$. In fact, making use of bifurcation theory and degree theory, we can solve this problem and further establish the multiplicity of positive solutions to (1.1) when $\gamma$ is suitably large. We first take $a$ as a bifurcation parameter and give the bifurcation solutions emanating from the semi-trivial solution $\left(0, \theta_{c}\right)$.

Theorem 3.3 Assume $c>\lambda_{1}$. Then $\left(0, \theta_{c} ; \lambda_{1}\left(\gamma \theta_{c}\right)\right)$ is a bifurcation point of the positive solution to (1.1). Moreover, there exist a constant $\delta>0$ and a $C^{1}$-curve $(a(s) ; U(s), V(s)):(0, \delta) \rightarrow$ $R \times X \times X$ such that

(i) $(U(s), V(s))$ is a positive solution of $(1.1)$ with $a=a(s)$ for each $s \in(0, \delta]$ and

$$
U(s)=s(\tilde{\Phi}+\Phi(s)), \quad V(s)=\theta_{c}+s(\tilde{\Psi}+\Psi(s))
$$

where $\tilde{\Phi}$ is the positive eigenfunction corresponding to $\lambda_{1}\left(\gamma \theta_{c}\right)$ with $\int_{\Omega} \tilde{\Phi}^{2} \mathrm{~d} x=1$, $\tilde{\Psi}=d \gamma\left(-\Delta-c+2 \theta_{c}\right)^{-1}\left(\theta_{c} \tilde{\Phi}\right)>0,(\Phi, \Psi) \in Z, Z \oplus \operatorname{span}\{(\tilde{\Phi}, \tilde{\Psi})\}=X \times X ;$

(ii) $a(0)=\lambda_{1}\left(\gamma \theta_{c}\right),(U(0), V(0))=\left(0, \theta_{c}\right)$, and $\Phi(0)=\Psi(0)=0$;

(iii) $a(s)$ has the derivative $a^{\prime}(0)=\rho(\gamma)$, where $\rho(\gamma)$ is defined by

$$
\rho(\gamma)=\gamma \int_{\Omega} \tilde{\Psi} \tilde{\Phi}^{2} \mathrm{~d} x-\frac{1}{2} \gamma^{2} \int_{\Omega} \theta_{c} \tilde{\Phi}^{3} \mathrm{~d} x
$$

Using the above theorem, we can obtain the following multiplicity result of positive solutions to (1.1).

Theorem 3.4 Assume that $c>\lambda_{1}$. Let $\gamma_{0}=2 \int_{\Omega} \tilde{\Psi} \tilde{\Phi}^{2} \mathrm{~d} x / \int_{\Omega} \theta_{c} \tilde{\Phi}^{3} \mathrm{~d} x$. If $\gamma>\gamma_{0}$, then there exists a constant $a_{*} \in\left(\lambda_{1}, \lambda_{1}\left(\gamma \theta_{c}\right)\right)$ such that (1.1) has at least two positive solutions for each $a \in\left(a_{*}, \lambda_{1}\left(\gamma \theta_{c}\right)\right)$ and has at least one positive solution for $a \geq \lambda_{1}\left(\gamma \theta_{c}\right)$.

Proof It follows from Theorem 3.2 that (1.1) has at least one positive solutions for each $a>\lambda_{1}\left(\gamma \theta_{c}\right)$. So we only have to show that (1.1) has at least two positive solutions for each $a \in\left(a_{*}, \lambda_{1}\left(\gamma \theta_{c}\right)\right)$ and has at least one positive solution for $a=\lambda_{1}\left(\gamma \theta_{c}\right)$.

Let $\mathcal{D}_{\epsilon}=\left\{(u, v) \in W:\left\|(u, v)-\left(0, \theta_{c}\right)\right\|_{E}<\epsilon\right\}$. Note that the direction of the bifurcation of (1.1) emanating from the semi-trivial solution $\left(0, \theta_{c}\right)$ is determined by the sign of $\rho(\gamma)$ given in (3.4). If $\gamma>\gamma_{0}$, then we see that $\rho(\gamma)<0$ and the bifurcation is subcritical. So there exists a positive constant $a_{*} \in\left(\lambda_{1}, \lambda_{1}\left(\gamma \theta_{c}\right)\right)$ such that for each $a \in\left(a_{*}, \lambda_{1}\left(\gamma \theta_{c}\right)\right),(1.1)$ has a unique positive solution $\left(u_{a}, v_{a}\right) \in \mathcal{D}_{\epsilon}$. To prove the existence of a positive solution when $a=\lambda_{1}\left(\gamma \theta_{c}\right)$ and two positive solutions when $a \in\left(a_{*}, \lambda_{1}\left(\gamma \theta_{c}\right)\right)$, it suffices to show that for each $a \in\left(a_{*}, \lambda_{1}\left(\gamma \theta_{c}\right)\right]$, (1.1) has a positive solution in $\mathcal{D}^{\prime} \backslash \mathcal{D}_{\epsilon}$.

Define $\mathcal{F}_{\tau}: \mathcal{D}^{\prime} \rightarrow W$ by

$$
\mathcal{F}_{\tau}(u, v)=(-\Delta+M)^{-1}\left(\begin{array}{c}
u(a-u)-\tau v\left(1-e^{-\gamma \theta_{a}}\right)+M u \\
v\left(c-v+\tau d\left(1-e^{-\gamma \theta_{a}}\right)\right)+M v
\end{array}\right),
$$

where $\tau \in[0,1], \mathcal{D}^{\prime}, W$ and $M$ are defined in Section 2. It follows from standard elliptic regularity theory that $\mathcal{F}_{\tau}$ is a completely continuous operator. Obviously, (1.1) has non-negative solutions if and only if the operator $\mathcal{F}_{1}$ has fixed points in $\mathcal{D}^{\prime}$. It is easy to 
check that $\mathcal{F}_{\tau}$ has no fixed point on $\partial\left(\mathcal{D}^{\prime} \backslash \mathcal{D}_{\epsilon}\right)$ for $\tau \in[0,1]$. Hence, index ${ }_{W}\left(\mathcal{F}_{\tau}, \mathcal{D}^{\prime} \backslash \mathcal{D}_{\epsilon}\right) \equiv$ constant. In particular, index ${ }_{W}\left(\mathcal{F}_{1}, \mathcal{D}^{\prime} \backslash \mathcal{D}_{\epsilon}\right)=\operatorname{index}_{W}\left(\mathcal{F}_{0}, \mathcal{D}^{\prime} \backslash \mathcal{D}_{\epsilon}\right)$. For small $\epsilon>0, \mathcal{F}_{0}$ has only three fixed points $(0,0),\left(\theta_{a}, 0\right)$ and $\left(\theta_{a}, \theta_{c}\right)$ in $\mathcal{D}^{\prime} \backslash \mathcal{D}_{\epsilon}$. It is well known that $\left(\theta_{a}, \theta_{c}\right)$ is linearly stable while $(0,0)$ and $\left(\theta_{a}, 0\right)$ are unstable, which implies $\operatorname{index}_{W}\left(\mathcal{F}_{0},\left(\theta_{a}, \theta_{c}\right)\right)=1$ and $\operatorname{index}_{W}\left(\mathcal{F}_{0},(0,0)\right)=\operatorname{index}_{W}\left(\mathcal{F}_{0},\left(\theta_{a}, 0\right)\right)=0$. Hence, we have

$$
\operatorname{index}_{W}\left(\mathcal{F}_{1}, \mathcal{D}^{\prime} \backslash \mathcal{D}_{\epsilon}\right)=\operatorname{index}_{W}\left(\mathcal{F}_{0}, \mathcal{D}^{\prime} \backslash \mathcal{D}_{\epsilon}\right)=0+0+1=1
$$

On the other hand, $\mathcal{F}_{1}$ has only two non-negative fixed points $(0,0)$ and $\left(\theta_{a}, 0\right)$ which are not positive in $\mathcal{D}^{\prime} \backslash \mathcal{D}_{\epsilon}$. By Lemma 2.5, both index ${ }_{W}\left(\mathcal{F}_{1},(0,0)\right)$ and $\operatorname{index}_{W}\left(\mathcal{F}_{1},\left(\theta_{a}, 0\right)\right)$ are zero. Thus one can assert that $\mathcal{F}_{1}$ has a fixed point in $\mathcal{D}^{\prime} \backslash \mathcal{D}_{\epsilon}$ other than $(0,0)$ and $\left(\theta_{a}, 0\right)$, which shows that (1.1) has a positive solution in $\mathcal{D}^{\prime} \backslash \mathcal{D}_{\epsilon}$. This completes the proof.

\section{The uniqueness of positive solutions}

The main result in this section is the following.

Theorem 4.1 Assume $\Omega=(p, q)$ for some real numbers $p<q$. If $\gamma^{2}(c+d) \leq 2$, then for every $(a, c)$ satisfying (3.3), the following boundary value problem:

$$
\begin{cases}-u^{\prime \prime}=u(a-u)-v\left(1-e^{-\gamma u}\right), & x \in(p, q), \\ -v^{\prime \prime}=\left(c-v+d\left(1-e^{-\gamma u}\right)\right) v, & x \in(p, q), \\ u(p)=u(q)=v(p)=v(q)=0 & \end{cases}
$$

has exactly one positive solution.

The proof will be finished in several steps. The technique we used here can be found in the papers $[17,18]$. The basic ingredient is non-degeneration of positive solutions, which is summarized as the following lemma.

Lemma 4.1 Let $\left(u_{*}, v_{*}\right)$ be an arbitrary positive solution of (4.1). Then the linearized problem of (4.1) at $\left(u_{*}, v_{*}\right)$,

$$
\begin{cases}-\phi^{\prime \prime}=\left(a-2 u_{*}-\gamma \nu_{*} e^{-\gamma u_{*}}\right) \phi-\left(1-e^{-\gamma u_{*}}\right) \psi, & x \in(p, q), \\ -\psi^{\prime \prime}=\left(c-2 v_{*}+d\left(1-e^{-\gamma u_{*}}\right)\right) \psi+d \gamma v_{*} e^{-\gamma u_{*}} \phi, & x \in(p, q), \\ \phi(p)=\phi(q)=\psi(p)=\psi(q)=0 & \end{cases}
$$

has only the trivial solution $(0,0)$. In other words, any positive solution is non-degenerate.

Proof Since $\left(u_{*}, v_{*}\right)$ is a positive solution of (4.1), by the Krein-Rutman theorem, we have

$$
\lambda_{1}\left(u_{*}+\frac{\nu_{*}\left(1-e^{-\gamma u_{*}}\right)}{u_{*}}-a\right)=\lambda_{1}\left(\nu_{*}-d\left(1-e^{-\gamma u_{*}}\right)-c\right)=0
$$

The linearized problem (4.2) can be written as

$$
\begin{cases}-\phi^{\prime \prime}+\left(2 u_{*}+\gamma v_{*} e^{-\gamma u_{*}}-a\right) \phi=-\left(1-e^{-\gamma u_{*}}\right) \psi, & x \in(p, q), \\ -\psi^{\prime \prime}+\left(2 v_{*}-d\left(1-e^{-\gamma u_{*}}\right)-c\right) \psi=d \gamma v_{*} e^{-\gamma u_{*}} \phi, & x \in(p, q), \\ \phi(p)=\phi(q)=\psi(p)=\psi(q)=0 . & \end{cases}
$$


From the monotonicity of $\lambda_{1}(\cdot)$, it follows that

$$
\lambda_{1}\left(2 \nu_{*}-d\left(1-e^{-\gamma u_{*}}\right)-c\right)>\lambda_{1}\left(v_{*}-d\left(1-e^{-\gamma u_{*}}\right)-c\right)=0 .
$$

If $\gamma^{2}(c+d) \leq 2$, then we claim that

$$
2 u_{*}+\gamma v_{*} e^{-\gamma u_{*}}>u_{*}+\frac{v_{*}\left(1-e^{-\gamma u_{*}}\right)}{u_{*}} .
$$

Thus

$$
\lambda_{1}\left(2 u_{*}+\gamma v_{*} e^{-\gamma u_{*}}-a\right)>\lambda_{1}\left(u_{*}+\frac{\nu_{*}\left(1-e^{-\gamma u_{*}}\right)}{u_{*}}-a\right)=0
$$

Now we shall prove that (4.4) is true. Let $h\left(u_{*}\right)=u_{*}^{2}+\gamma u_{*} \nu_{*} e^{-\gamma u_{*}}-v_{*}\left(1-e^{-\gamma u_{*}}\right)$. It suffices to show $h\left(u_{*}\right)>0$. Obviously, $h(0)=0$ and $h^{\prime}\left(u_{*}\right)=u_{*}\left(2-\gamma^{2} v_{*} e^{-\gamma u_{*}}\right)$. Reminding $v_{*}<c+d$ given by Lemma 2.2, we get $h^{\prime}\left(u_{*}\right)>0$ and thus $h\left(u_{*}\right)>0$. This shows that (4.4) holds true.

Define the operators $L_{1}$ and $L_{2}$ by

$$
\begin{cases}L_{1} \phi=-\psi^{\prime \prime}+\left(2 u_{*}+\gamma v_{*} e^{-\gamma u_{*}}-a\right) \phi, & \phi \in X, \\ L_{2} \psi=-\psi^{\prime \prime}+\left(2 v_{*}-d\left(1-e^{-\gamma u_{*}}\right)-c\right) \psi, & \psi \in X .\end{cases}
$$

So (4.2) can be written as

$$
L_{1} \phi=-\left(1-e^{-\gamma u_{*}}\right) \psi, \quad L_{2} \psi=d \gamma v_{*} e^{-\gamma u_{*}} \phi .
$$

By (4.3) and (4.5), $L_{1}$ and $L_{2}$ have inverses, say $L_{1}^{-1}, L_{2}^{-1}$, which are compact and orderpreserving, i.e., $L_{i}^{-1}(P-\{0\}) \subset \operatorname{int} P$ for $i=1,2$. Now we shall show that the only solution to $(4.6)$ is $(0,0)$, which completes the proof of this lemma. To this end, we argue by contradiction, assuming that there exists a solution $(\phi, \psi) \neq(0,0)$ to $(4.2)$. From (4.6), it follows that

$$
-\phi=L_{1}^{-1}\left[\left(1-e^{-\gamma u_{*}}\right) L_{2}^{-1}\left(d \gamma \nu_{*} e^{-\gamma u_{*}} \phi\right)\right] .
$$

Since the right-hand side of (4.7) defines a compact strongly order-preserving operator, we find that $\phi$ must change sign in $(p, q)$. Similarly, $\psi$ must change sign in $(p, q)$. Moreover, $\phi$ and $\psi$ cannot vanish on an interval of positive length by the maximum principle, i.e., the zeros of $\phi, \psi$ are isolated each from the others. In fact, if $\phi$ vanishes on an interval, then $\phi^{\prime}=0$ at the boundary of such an interval where $\phi>0$ or $\phi<0$, which contradicts the maximum principle. Thus there exists a partition of $(p, q)$, say

$$
Q=\left\{p=x_{0}<x_{1}<x_{2}<\cdots<x_{n-1}<x_{n}=q\right\}
$$

and we can choose $\phi$ such that

$$
\begin{aligned}
& \phi(x)>0, \quad x \in\left(x_{2 k}, x_{2 k+1}\right), k \geq 0,2 k+1 \leq n, \\
& \phi(x)<0, \quad x \in\left(x_{2 k-1}, x_{2 k}\right), k \geq 1,2 k \leq n, \\
& \phi(x)=0, \quad x=x_{k}, 0 \leq k \leq n .
\end{aligned}
$$


Here we claim that

$$
\psi\left(x_{2 k}\right)>0, \quad \psi\left(x_{2 k+1}\right)<0, \quad x_{2 k}, x_{2 k+1} \in Q-\{p, q\} .
$$

Since the principal eigenvalues of $L_{1}$ and $L_{2}$ on any subinterval of $(p, q)$ are strictly positive, the generalized maximum principle will be used to show this claim. By hypothesis, $\phi(x)>$ $0, x \in\left(x_{0}, x_{1}\right)$ and $\phi\left(x_{0}\right)=\phi\left(x_{1}\right)=0$. Thus

$$
L_{2} \psi(x)=d \gamma v_{*} e^{-\gamma u_{*}} \phi(x)>0, \quad x \in\left(x_{0}, x_{1}\right) .
$$

We claim that $\psi\left(x_{1}\right)<0$. In fact, if $\psi\left(x_{1}\right) \geq 0$, then by the generalized maximum principle, we have $\psi(x)>0, x \in\left(x_{0}, x_{1}\right)$. Thus

$$
L_{1} \phi(x)=-\left(1-e^{-\gamma u_{*}}\right) \psi(x)<0, \quad x \in\left(x_{0}, x_{1}\right)
$$

Therefore $\phi(x)<0, x \in\left(x_{0}, x_{1}\right)$, which contradicts (4.8). So $\psi\left(x_{1}\right)<0$.

Again by hypothesis, $\phi(x)<0, x \in\left(x_{1}, x_{2}\right)$ and $\phi\left(x_{1}\right)=\phi\left(x_{2}\right)=0$. Thus

$$
L_{2} \psi(x)=d \gamma v_{*} e^{-\gamma u_{*}} \phi(x)<0, \quad x \in\left(x_{1}, x_{2}\right) .
$$

We claim that $\psi\left(x_{2}\right)>0$. In fact, if $\psi\left(x_{2}\right) \leq 0$, then by the generalized maximum principle, we have $\psi(x)<0, x \in\left(x_{1}, x_{2}\right)$. Thus

$$
L_{1} \phi(x)=-\left(1-e^{-\gamma u_{*}}\right) \psi(x)>0, \quad x \in\left(x_{1}, x_{2}\right) .
$$

Therefore $\phi(x)>0, x \in\left(x_{1}, x_{2}\right)$, which contradicts (4.8). So $\psi\left(x_{2}\right)>0$. Arguing recursively, we show (4.9) holds.

According to the parity of $n$, either

$$
\phi(x)>0, \quad x \in\left(x_{2 k}, q\right), \quad \psi\left(x_{2 k}\right)>0
$$

or

$$
\phi(x)<0, \quad x \in\left(x_{2 k+1}, q\right), \quad \psi\left(x_{2 k+1}\right)<0
$$

is satisfied. Assume (4.10) holds. Then

$$
L_{2} \psi(x)=d \gamma v_{*} e^{-\gamma u_{*}} \phi(x)>0, \quad x \in\left(x_{2 k}, q\right) .
$$

Since $\psi\left(x_{2 k}\right)>0$ and $v(q)=0$, by the generalized maximum principle, we have $\psi(x)>0$, $x \in\left(x_{2 k}, q\right)$. Thus

$$
L_{1} \phi(x)=-\left(1-e^{-\gamma u_{*}}\right) \psi(x)<0, \quad x \in\left(x_{2 k}, q\right) .
$$

Therefore $\phi(x)<0, x \in\left(x_{2 k}, q\right)$, which contradicts (4.8). Similarly, if (4.11) holds, we also get a contradiction. The proof of Lemma 4.1 is complete. 
Applying the implicit function theorem, we can show that if (4.1) has exactly one positive solution, which in addition is non-degenerate, then the following problem:

$$
\begin{cases}-u^{\prime \prime}=u(a-u)-v\left(1-e^{-(\gamma+\epsilon) u}\right), & x \in(p, q), \\ -v^{\prime \prime}=\left(c-v+d\left(1-e^{-(\gamma+\epsilon) u}\right)\right) v, & x \in(p, q), \\ u(p)=u(q)=v(p)=v(q)=0 & \end{cases}
$$

has also exactly one positive solution provided $\epsilon$ is small enough. The proof is omitted.

Lemma 4.2 Suppose that (3.3) is satisfied and (4.1) has exactly one positive solution $\left(u_{*}, v_{*}\right)$, which is non-degenerate. Then there exists $\epsilon_{0}=\epsilon(a, c, d, \gamma)>0$ such that for every $\epsilon \in\left(-\epsilon_{0}, \epsilon_{0}\right)$ the problem (4.12) has exactly one positive solution $(u(\epsilon), v(\epsilon))$. Moreover, $(u(0), v(0))=\left(u_{*}, v_{*}\right)$ and the mapping $\epsilon \rightarrow(u(\epsilon), v(\epsilon))$, from a neighborhood of $\epsilon=0$ in $R$ to $X^{2}$, belongs at least to the class $C^{1}$.

Proof of Theorem 4.1 Consider the set

$$
\Gamma:=\{\hat{\gamma} \in[0, \gamma]:(4.1) \text { with } \gamma=\beta \text { has a unique positive solution, } \forall \beta \in[0, \hat{\gamma}]\} \text {. }
$$

Since (4.1) with $\gamma=0$ is uncoupled, if it has a positive solution, then it has exactly one. Thus $0 \in \Gamma$, i.e., $\Gamma$ is not empty. By Lemma 4.2 , we know that $\Gamma$ is open in $[0, \gamma]$. Now we shall show that $\Gamma$ is closed in $[0, \gamma]$. Hence $\Gamma=[0, \gamma]$, which completes the proof. To show this, consider a sequence $\left\{\gamma_{n}\right\}_{n \geq 1}$ in $\Gamma$ satisfying $\gamma_{n} \rightarrow \tilde{\gamma} \leq \gamma$ as $n \rightarrow \infty$. Since the mapping $\gamma \rightarrow \lambda_{1}\left(\gamma \theta_{c}\right)$ is increasing in $\gamma$, both (3.3) and $\gamma^{2}(c+d) \leq 2$ are satisfied for $\gamma=\tilde{\gamma}$ and $\gamma=\gamma_{n}, n \geq 1$. Let $\left(u_{n}, v_{n}\right)$ be the unique positive solution of (4.1) with $\gamma=\gamma_{n}$. By passing to a subsequence if necessary, $\left(u_{n}, v_{n}\right)$ converges to $(\tilde{u}, \tilde{v})$ as $n \rightarrow \infty$. From the proof of Theorem 3.2, it follows that $(\tilde{u}, \tilde{v})$ is in the interior of $P \times P$. Since (3.3) is satisfied for $\gamma=\tilde{\gamma}$, we know that $(\tilde{u}, \tilde{v})$ is a positive solution of (4.1) with $\gamma=\tilde{\gamma}$. In fact, the problem (4.1) with $\gamma=\tilde{\gamma}$ has exactly one positive solution. Otherwise the application of the implicit function theorem would imply that (4.1) with $\gamma=\gamma_{n}$ has at least two positive solutions for sufficiently large $n$, contradicting the fact that $\gamma_{n} \in \Gamma$. Hence $\tilde{\gamma} \in \Gamma$ and $\Gamma=[0, \gamma]$. This finishes the proof.

\section{Competing interests}

The authors declare that they have no competing interests.

\section{Authors' contributions}

GG performed the theory analysis and carried out some computations. BL participated in the sequence alignment and also undertook some computations. XL participated in the design of the study. All authors read and approved the final manuscript.

\section{Author details}

'College of Science, Shaanxi University of Science and Technology, Xi'an, 710021, P.R. China. ${ }^{2}$ Department of Basic Course, Shaanxi Railway Institute, Weinan, 714000, P.R. China.

\section{Acknowledgements}

The work is supported by the Natural Science Foundation of China (Nos. 11271236, 11001160), the Natural Science Basic Research Plan in Shaanxi Province of China (No. 2011JQ1015) and the Foundation of Shaanxi Educational Committee of China (No. 12JK0856). 


\section{References}

1. Ivlev, V: Experimental Ecology of the Feeding Fishes. Yale University Press, New Haven (1961)

2. Kooij, RE, Zegling, A: A predator-prey model with Ivlev's functional response. J. Math. Anal. Appl. 198, 473-489 (1996)

3. Sugie, J: Two-parameter bifurcation in a predator-prey system of Ivlev type. J. Math. Anal. Appl. 217, 349-371 (1998)

4. Baek, HK, Kim, SD, Kim, P: Permanence and stability of an Ivlev-type predator-prey system with impulsive control strategies. Math. Comput. Model. 50, 1385-1393 (2009)

5. Wang, HL, Wang, WM: The dynamical complexity of a Ivlev-type prey-predator system with impulsive effect. Chaos Solitons Fractals 38, 1168-1176 (2008)

6. Xiao, HB: Global analysis of Ivlev's type predator-prey dynamic systems. Appl. Math. Mech. 38, 461-470 (2007)

7. Ling, L, Wang, WM: Dynamics of a Ivlev-type predator-prey system with constant rate harvesting. Chaos Solitons Fractals 41, 2139-2153 (2009)

8. Wang, WM, Zhang, L, Wang, HL, et al.: Pattern formation of a predator-prey system with Ivlev-type functional response. Ecol. Model. 221, 131-140 (2010)

9. Jia, YF: A sufficient and necessary condition for the existence of positive solutions for a prey-predator system with Ivlev-type functional response. Appl. Math. Lett. 24, 1084-1088 (2011)

10. Smoller, J: Shock Waves and Reaction-Diffusion Equations. Springer, New York (1983)

11. Dancer, EN: On the indices of fixed points of mappings in cones and applications. J. Math. Anal. Appl. 91, 131-151 (1983)

12. Cassanova, CS: Existence and structure of the set of positive solutions of a general class of sublinear elliptic non-classical mixed boundary value problems. Nonlinear Anal. TMA 49(3), 361-430 (2002)

13. Crandall, MG, Rabinowitz, PH: Bifurcation from simple eigenvalues. J. Funct. Anal. 8(2), 321-340 (1971)

14. Yamada, Y: Stability of steady states for prey-predator diffusion equations with homogeneous Dirichlet conditions. SIAM J. Math. Anal. 21, 327-345 (1990)

15. Guo, GH, Wu, JH: Multiplicity and uniqueness of positive solutions for a predator-prey model with B-D functional response. Nonlinear Anal. TMA 72, 1632-1646 (2010)

16. Blat, J, Brown, KJ: Global bifurcation of positive solutions in some systems of elliptic equations. SIAM J. Math. Anal. 17, 1339-1353 (1986)

17. López-Gómez, J, Pardo, R: Existence and uniqueness of coexistence states for the predator-prey Lotka-Volterra model with diffusion on intervals. Differ. Integral Equ. 6, 1025-1031 (1993)

18. Casal, A, Eilbeck, JC, López-Gómez, J: Existence and uniqueness of coexistence states for a predator-prey model with diffusion. Differ. Integral Equ. 7, 411-439 (1994)

doi:10.1186/1687-1847-2013-164

Cite this article as: Guo et al.: Qualitative analysis on a predator-prey model with Ivlev functional response. Advances in Difference Equations 2013 2013:164.

\section{Submit your manuscript to a SpringerOpen ${ }^{\circ}$ journal and benefit from:}

- Convenient online submission

- Rigorous peer review

- Immediate publication on acceptance

- Open access: articles freely available online

- High visibility within the field

- Retaining the copyright to your article 